\title{
Service assignment decision support of one-way carsharing in wireless charging road network
}

\author{
Yixi Liu ${ }^{1 *}$ \\ ${ }^{1}$ School of Traffic and Transportation, Beijing Jiaotong University, Beijing, 100044, People's Republic of China
}

\begin{abstract}
Once wireless charging lanes are commercialized, vehicles can be charged during the dispatch process, which is of great value to the electrification of one-way car-sharing fleet. In the city road network occupied with wireless charging lanes, service order arrangement of a car tends to select stations to make full use of the dispatch process for charging and improve the efficiency of vehicle circulation. The combination of wireless charging lanes and site charging provides a new perspective for service decision-making: scheduling from different alternative sites to sites lack of cars causes different power consumption or increase, charging costs, and travel time. Decision makers need to weigh these factors in order to get more profit, which puts forward new requirements for the service connection and dispatch of one-way shared cars.
\end{abstract}

\section{Introduction}

Among the related research on car-sharing in recent years, the charging behavior of electric vehicles is an important content. As wireless charging technology develops rapidly, the construction and application of wireless charging is on the road [1]. The deployment of wireless charging lanes (WCL) on urban roads not only sheds lights on alleviating the difficulty of charging electric vehicles, but also provides possibility to improve car-sharing operation. At the same time, the proportion of charging facilities in urban public parking lots is gradually increasing. Therefore, we focus on the one-way car-sharing service selection based on the cooperation of both station charge and lane charge.

In the car-sharing field, the application of wireless charging can make it easier to relieve users' mileage anxiety and also share station charging pressure, reduce the station charging time of the vehicle, thus improve the efficiency of the vehicle usage. For these reasons, it's is beneficial for the development of car-sharing operation. Reference [2] gives an overview of recent studies about one-way car-sharing, and there is no such research to explore it under the background of WCL in city. To this end, we address the problem of optimizing one-way carsharing service combined with wireless charging lanes located.

At present, some relevant research on wireless charging has emerged. Jang provides an overview of recent studies about wireless charging and concluded that the current research mainly includes several aspects in [3], such as wireless charging infrastructure allocation, pricing policy like in [4], cost and benefit analysis and other decision supporting like [5]. He et al. considered its impact on the traffic capacity of the road network and the route selection of travelers in the WCL location optimization decision and established a nonlinear model in [6]. Chen et al. made joint decisions on the optimal configuration of charging stations on both sides of the traffic corridor and wireless lane charging based on travel demand in [7], and demonstrated the competitive advantage of dynamic wireless charging in certain situations.

Given that it's promise for WCL infrastructure and site charging facilities to contribute to vehicle charging jointly in one-way car-sharing, car-sharing industry may face a new situation and the scheduling decision will present new characteristics. This paper focuses on studying the impacts of WCL on one-way car-sharing operation decision in the car-sharing service assignment and relocation model.

\section{Materials and Methods}

When wireless charging lanes and site charging are combined, it leads to a new discuss of demand dispatch in one-way car-sharing system. Scheduling vehicles for the purpose of charging will reflect on the selection of relocation stations. To maximize its profit, the operator may choose stations to help EVs charging. When selecting a call-out station(station with idle cars), it should also consider whether the route between the alternative call-out site and the call-in site(station lack of cars) can supplement the vehicle power, how much power can be supplemented, the travel time and other cost factors to make the overall vehicle scheduling decision. To gain more insight into this problem, we conducted an 0-1 mixed integer model in the following section.

\subsection{Problem Define}

On the road network equipped with wireless charging lanes, the car-sharing sites located at the road section 
nodes are rent in advance, and all parking plots are equipped with charging facilities. The car-sharing fleet are homogeneous vehicles, that is, the charge and discharge performance of all vehicles is the same.

\subsection{Assumptions:}

- We adopt a full charge strategy, that is, the battery is fully charged before each departure.

- All orders are assumed to be known. The information includes start station, namely $\mathrm{O}$, end station, namely $\mathrm{D}$, departure time at $\mathrm{O}$, arrival time at $\mathrm{D}$ calculated by on the shortest path, and power consumption or supplement on the road.

- Assume that the vehicle charging rate is the same, and the power consumption rate is the same $\beta$. Charge and power consumption are related to time. Dispatching should consider the time window of customer demand and the different charging time required for dispatching vehicles for different order sequences.

- The travel time of each path is fixed to the shortest path, and the charging amount of the wireless charging lane is related to links travel time. We mail focus on car-sharing cars and do not take road congestion into account.

- The initial number of vehicles at each station is known.

- The site is located at a node of the road network, and the length of time and the amount of charging/power consumption of the road section between each node adopt the shortest path.

- Both lane charging and site charging are available.

\subsection{Notations}

Table1. Notations and Definitions

\begin{tabular}{|c|c|c|}
\hline \multirow{2}{*}{ Notations } & \multicolumn{2}{|c|}{ Definitions and units } \\
\cline { 2 - 3 } & Definition & Unit \\
\hline $\mathrm{H}$ & The set of electric cars & - \\
\hline $\mathrm{N}$ & The set of stations & - \\
\hline $\mathrm{K}$ & The set of orders & - \\
\hline $\mathrm{pt}$ & $\begin{array}{c}\text { Service charge price for } \\
\text { carsharing }\end{array}$ & Yuan(min) \\
\hline$\alpha 1$ & electricity recharge rate of WCL & $\mathrm{kwh}(\mathrm{min})$ \\
\hline$\alpha 2$ & electricity recharge rate of station & $\mathrm{kwh}(\mathrm{min})$ \\
\hline$\beta$ & electricity consumption rate & $\mathrm{kwh}(\mathrm{min})$ \\
\hline $\mathrm{Cs}$ & electricity recharge fee of station & Yuan(min) \\
\hline $\mathrm{Cl}$ & electricity recharge fee of WCL & Yuan(min) \\
\hline $\mathrm{t}_{\mathrm{s} \mathrm{j}}$ & $\begin{array}{c}\text { Travel time to relocate cars to } \\
\text { serve order } \mathrm{j}\end{array}$ & $\mathrm{min}$ \\
\hline $\mathrm{r}_{\mathrm{s} j}$ & $\begin{array}{c}\text { Travel cost to relocate cars to } \\
\text { serve order } \mathrm{j}\end{array}$ & $\mathrm{Yuan}$ \\
\hline $\mathrm{n}_{\mathrm{h}}$ & $\begin{array}{c}\text { The initial parking station of } \\
\text { vehicle } h\end{array}$ & - \\
\hline
\end{tabular}

For each $\mathrm{j}$ in $\mathrm{K}$, the related demand information is expressed as $\left\{O_{j}, D_{j}, O t_{j}, D t_{j}, b_{j}, p_{j}, c_{j}\right\}$, where $O_{j}$ is the start station of order $\mathrm{j}, D_{j}$ is the end station of order $\mathrm{j}, O t_{j}$ is the start time of order $\mathrm{j}$, and $D t_{j}$ the end time for the shortest path between $\mathrm{O}$ and $\mathrm{D}$. The value of $D t_{j}, b_{j}, p_{j}, c_{j}$ are calculated on the basis of the travelling time and road charging time decided by shortest path rule. For example, when a vehicle pass link a on its relocation route, $\alpha 1$ is the amount of power received per unit time from the WCL charging pads, $\beta$ is the electricity consumption rate, the time cost $t_{j}$ is the sum of all the links that cars pass through to cover the shortest path. Similarly $t e_{j}$ is the sum travel time of links with charging pads. $D t_{j}$ equals $O t_{j}$ plus $t_{j}$, and $p_{j}$ the product of $p t$ multiply by $t_{j}$. The electric power consume of demand $\mathrm{j}$ is denote with $b_{j}$.

\subsection{Equations}

The service dispatch model can be formulated as follows

$$
\max Z=\sum_{h=1}^{H} \sum_{i=1}^{K} \sum_{j=1}^{K} x_{s j}^{h}\left(p_{j}-r_{s j}-C s\left(c b t_{j}+c a t_{j}\right)\right.
$$

Equation (1) is to maximize the daily profit of carsharing operator, $x_{s j}^{h}$ indicates two different situations: for $\mathrm{s}=\mathrm{i}, x_{i j}^{h}$, equals 1 if a vehicle $\mathrm{h}$ is assigned to serve order $\mathrm{j}$ after order $\mathrm{i}$ and 0 otherwise; while for $\mathrm{s}=0, x_{0 j}^{h}$ equals 1 if a vehicle $h$ is assigned to serve order $j$ as its first task. $p_{j}$ is the rent of order $\mathrm{j}$ paid by its user, $r_{s j}$ the travelling cost where $\mathrm{s}$ is the same as in $x_{s j}^{h}$. The third term in (1) is the total cost at car-sharing station, namely to multiply recharge fee of station $C s$ by the sum value of $c b t_{j}$ the charge time before order $\mathrm{j}$ and $c a t_{j}$ the charge time after order $\mathrm{j}$.

For each order arranged into the serve sequence, it is part of the flow balance as in (2), $r s_{j}$ is the relocation departure time from the end station of order $\mathrm{j}$.

$$
\begin{array}{r}
\sum_{h=1}^{H} \sum_{s=0}^{K} x_{s j}^{h}-\sum_{h=1}^{H} \sum_{s=0}^{K} x_{j s}^{h}=0, \forall j \in K \\
\sum_{h=1}^{H} \sum_{s=0}^{K} x_{s j}^{h} \leq 1, \forall j \in K \\
\sum_{s=0}^{K} x_{s j}^{h} \leq 1, \forall h \in H \\
x_{i j}^{h}+x_{j i}^{h} \leq 1, \forall i, j \in K
\end{array}
$$




$$
\begin{gathered}
\sum_{i=1}^{K} x_{j i}^{h} \leq \sum_{s=0}^{K} x_{s j}^{h}, \forall h \in H, j \in K \\
x_{0 j}^{h} t_{0 j} \leq O t_{j}, \forall j \in K, h \in H \\
\sum_{h=1}^{H} x_{i j}^{h}\left(r s_{i}+t_{i j}\right) \leq O t_{j}, \forall \mathrm{i}, \mathrm{j} \in K \\
r s_{i}+t_{i j} \leq \sum_{h=1}^{H} x_{i j}^{h} O t_{j}+M\left(1-\sum_{h=1}^{H} x_{i j}^{h}\right), \forall i, j \in K \\
r s_{j} \geq D t_{j}+c a t_{j}, \forall j \in K \\
c b t_{j}+r s_{i}+t_{i j}+M\left(\sum_{h=1}^{H} x_{i j}^{h}-1\right) \leq O t_{j}, \forall i, j \in K \\
\alpha b t_{j}, c a t_{j}, r s_{j} \in \mathbb{N}, \forall j \in K \\
\alpha 2 c a t_{j} \geq \sum_{h=1}^{H} \sum_{s=0}^{K} x_{s j}^{h} b_{j}, \forall j \in K \\
x_{s j}^{h} \in\{0,1\}, \forall s \in S, j \in K, h \in H \\
\sum_{h=1}^{H} x_{i j}^{h} D t_{j}+c a t_{j}-M\left(1-\sum_{h=1}^{H} \sum_{s=0}^{K} x_{s j}^{h} b_{s j}, \forall i, j \in K\right. \\
\left.x_{h=1}^{H} x_{i j}^{h}\right) \leq r s_{j}, \forall i, j \in K
\end{gathered}
$$

Each order is only in one sequence for at most one time as described in (3); Equation (4) ensures each car serves up to one demand one time; Users sequence is not a circle as in (5); Equation (6) indicates that for any vehicle h, only when it has served user $j$, can it go to serve the next user of $j$; The constraints about the service order are presented in (7) to (8) and (8) is linearizes as (9); if demand $\mathrm{j}$ is chosen, the travel time to relocate cars to its start point $t_{s j}$ needs to be lower than the time gap. Time gap here is expressed by its start time minus the expected departure time from the previous order end station.

Other time constraints are presented by (10), (11) and (13). Equation (10) is used to describe the relationship of $r s_{j}$ and $c a t_{j}$. One car need to complete charge before its departure from $D_{j}$. In (11), if $\sum_{h=1}^{H} x_{i j}^{h}$ equals 1 , the charge time before order $\mathrm{j} c b t_{j}$ plus $r s_{i}$ the relocation departure time from the end station of order i plus $t_{i j}$ must be earlier than the prescribed departure time $O t_{j}$. Equation (13) is built in the same way as (11). The charge time at station is calculated by constraints (12) to (14). Finally, variable value range is defined in (15) and (16).

\section{Results \& Discussion}

The following section shows some numerical examples based on Nguyen-Dupuis network to demonstrate the effectiveness of the proposed model and analyze WCL's effect on car-sharing operation. We solved the mixed integer linear programming model by CPLEX optimization studio 12.6.

\subsection{The Nguyen-Dupuis network}

In this paper, we adopt The Nguyen-Dupuis network which contains 13 nodes and 19 links. All the nodes on this network are rent by car-sharing company in advance and the travel time of every neighboring point pair are labeled on the link.

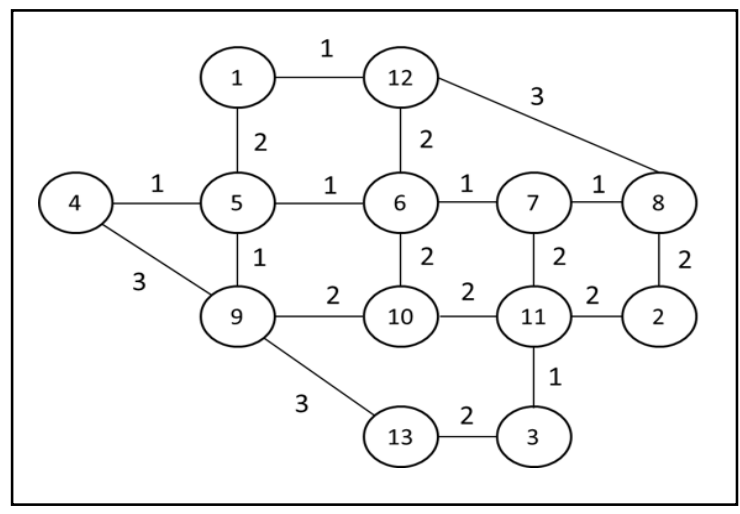

Figure 1. Links Labeled Nguyen-Dupuis network

We split the operation time into 30 equal periods referred to as $\mathrm{T}$, each $\mathrm{T}$ contains 30 minutes. Travel time of each link is shown by the number of $\mathrm{T}$ it costs. The Demand data set is generated randomly, where the computer selects $O_{j}, D_{j}$ and $O t_{j}$ by uniform distribution for each demand record $\mathrm{j}$. The WCL charge rate (power received from $\mathrm{WCL}$ ) is set to be $3 \mathrm{kwh} / \mathrm{min}$ as suggested in [1], station rate $3 \mathrm{kwh} / \mathrm{min}$ and power consume rate $1.5 \mathrm{kwh} / \mathrm{min}$.

\subsection{Numerical Examples}

First, we explore the impact of charging lane length on the performance of car-sharing. Fig. 1 Shows that with the decline of the WCL section, the profit and demand service volume are declining stepwise. Only 2/32 length proportion of WCL may cause the profit to increase more than $3 \%$. When the total mileage of the charging section is $20 / 32$, the profit and demand service volume get close to the best value. Profit and demand service volume indicators have almost no change after that point. 


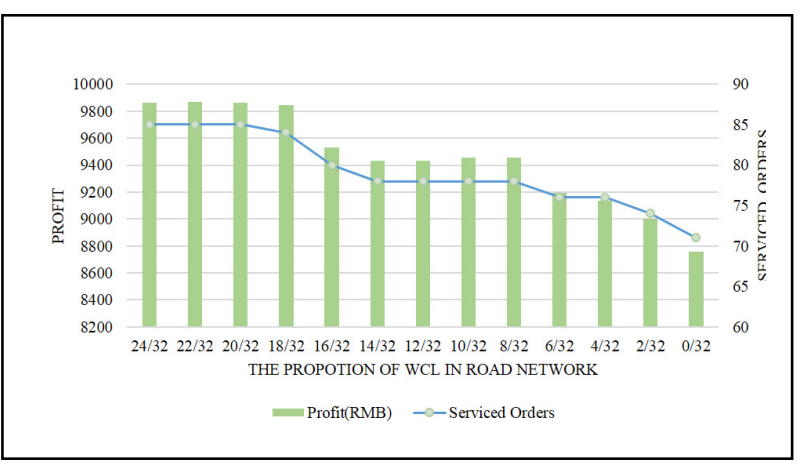

Figure 2. Profit and service level for different WCL length.

As we can see in Fig. 2, the wireless charging lane configuration in the vicinity of this proportion has been able to fully utilize the advantages of the wireless charging lane.

In the following section, the WCL length proportion is fixed to show the change of charging fee's inflection on car-sharing profit.

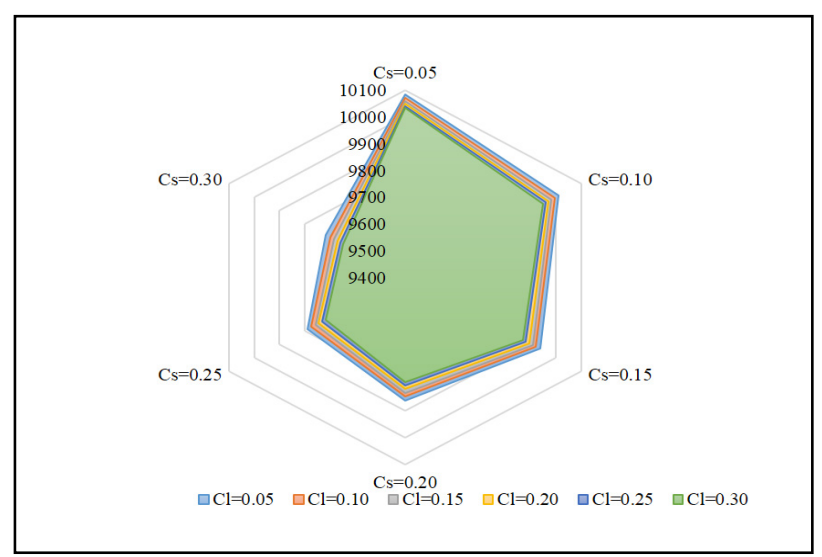

Figure 3. Profit performance for WCL and station charge fee.

The best charging lane configuration adopted, the series in Fig. 3 indicate the charging fees for different road sections. With the increase in unit site charging cost, the overall profit change slightly, but the overall trend is that as the site charging costs increase, the profit gap corresponding to the charging costs of different road sections increases. When the site charging fee reaches 0.30 yuan per minute, the profit reduction is most significant.

When the optimal charging lane length is in configuration, as the charging cost of the site increases, the service level is basically unchanged. At this time, most of the demand has a higher utilization rate of the charging lane and low dependence on the charging of the site. After the site fee Cs was adjusted to 0.25 yuan per minute, the serviced number for orders decreases. Figure 4 shows that once the charge exceeding 0.25 yuan/minute, it may have a greater impact on the cost, leaving some demand unserviced due to the high cost.

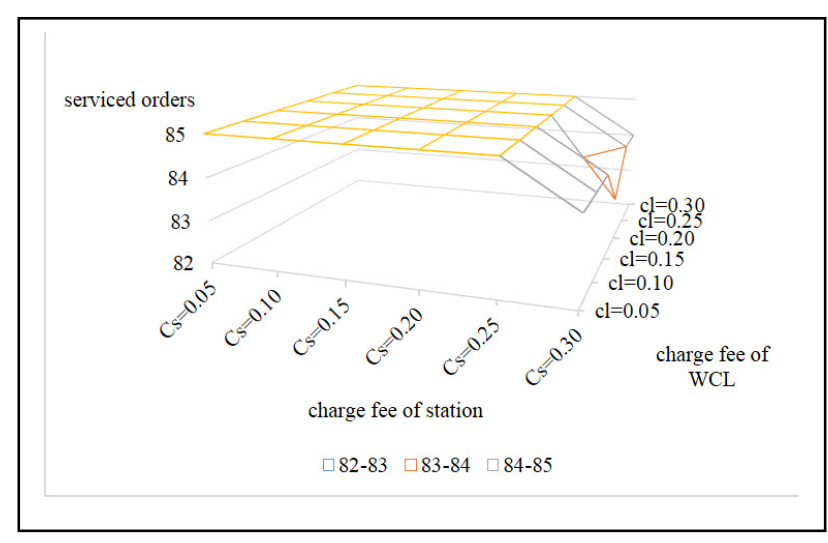

Figure 4. Service performance for WCL and station charge fee

\section{Conclusion}

In this paper, we study the problem of how to allocate carsharing cars to serve users demand on a road network with WCL. We cope with the service order decision plan analyzed the influence of WCL on car-sharing system by proposing a $0-1 \mathrm{mix}$ integer programming model. The numerical examples show that city road embedded with WCL can improve car-sharing operation benefit up to $10 \%$, which proves that wireless charging lane has a positive effect on the operation of shared cars.

\section{References}

1. Fuller, M., 2016. Wireless charging in California: Range, recharge, and vehicle electrification. Transp. Res. Part C 67, 343-356.

2. Illgen, S. and M. Hock, 2019. Literature review of the vehicle relocation problem in one-way car sharing networks. Transportation Research Part BMethodological 120: 193-204.

3. Jang, Y.J., 2018. Survey of the operation and system study on wireless charging electric vehicle systems. Transp. Res. Part C 95, 844-866.

4. Helber S, Broihan J, Jang YJ, Hecker P, Feuerle T.,2018. Location Planning for Dynamic Wireless Charging Systems for Electric Airport Passenger Buses. Energies. 11(2):258

5. Park, J.H., Jeong, Y.H., 2017. The Economics of Wireless Charging on the Road, The On-line Electric Vehicle. Springer pp. 329-345.

6. He, J., Yang, H., Tang, T.Q., Huang, H.J., 2018. An optimal charging station location model with the consideration of electric vehicle's driving range. Transp. Res. PartC 86, 641-654.

7. Chen, Z., Liu, W., Yin, Y., 2017. Deployment of stationary and dynamic charging infrastructure for electric vehicles along tra\#c corridors. Transport. Res. Part C:Emerg. Technol. 77, 185-206. 